\title{
Mehr Licht im Chemieunterricht! Experimentelle Zugänge zu Grundkonzepten der Photochemie
}

\author{
Michael W. Tausch*
}

\begin{abstract}
On September 8, 2016, the Swiss Chemical Society (SCS) organized a symposium called the 'Future of Chemical Education' with lectures and workshops during the SCS Fall meeting at ETH Zurich. This article is a summary of the lecture and workshop that the author held at this event in which teachers carried out model experiments on photocatalysis and conversion of solar energy into stored chemical energy.
\end{abstract}

Keywords: angeregte Zustände · curriculare Innovationsforschung · Nachhaltigkeit · Photoprozesse

\section{Warum „Mehr Licht im Chemieunterricht"?}

Diese Frage ist angesichts der vollen, oft übervollen Lehrpläne für den Chemieunterricht berechtigt. Es gibt aber kaum ein Schulfach, dessen Inhalte sich so rasch erneuern wie in der Chemie. Dabei geht es nicht um die Grundkonzepte dieser Disziplin, wohl aber um die Beispiele, an denen sie im Unterricht erschlossen werden. In diesem Zusammenhang sind Alltagsphänomene, technische Anwendungen und Zukunftsvisionen, bei denen Licht eine zentrale Rolle spielt, von einer herausragenden Bedeutung.

Unterdenglobalen Herausforderungen des 21. Jahrhunderts nimmt die Energieversorgung bei wachsender Erdbevölkerung und steigendem durchschnittlichem Energiebedarf pro Erdbewohner einen Sonderplatz ein. Die Bereitstellung von Energie für die durch Technik geprägte Zivilisation muss gleichzeitig kostengünstig und langfristig sicher, umweltschonend und nachhaltig sein. Die Sonneneinstrahlung beträgt in jedem Jahr das 100-fache der gesamten als abbaufähig erachteten Weltreserven an Kohle,

\footnotetext{
${ }^{\star}$ Correspondence: Prof. Dr. M. W. Tausch

Bergische Universität Wuppertal

Fakultät 4, Chemie, Gaußstr. 20

D-42119 Wuppertal

E-Mail: mtausch@uni-wuppertal.de
}

Erdöl, Erdgas und Uran. ${ }^{[1]}$ Der Mensch hat in den letzten Jahrhunderten des ,anthropocene" die natürlichen Bedingungen für sein langfristiges Überleben auf diesem Planeten massiv beeinträchtigt. Er hat zum einen riesige, Photosynthese treibende Waldflächen vernichtet und zum anderen Kohlenstoff aus den fossilen chemischen Energiespeichern als Kohlenstoffdioxid in die Atmosphäre gebracht. Dadurch hat er die stoffliche Bilanz beim Kreislauf des Kohlenstoffs und seiner Verbindungen in doppelter Weise zuungunsten der Nutzung von Solarlicht beim energetischen Antrieb der Biosphäre beeinflusst. Im ,sustainocene ${ }^{\text {" }}{ }^{[2]}$ der neuen Ära, in die sich die Menschheit begibt, muss das Prinzip der Nachhaltigkeit Vorrang erhalten. Das bedeutet, dass auch die Technosphäre ebenso wie die aus der natürlichen Evolution hervorgegangene Biosphäre vorwiegend die Energieform nutzt, die dem Planeten in kosmischen Größenordnungen und über astronomische Zeiträume hinweg zur Verfügung steht und darüber hinaus, aus der Sicht der höheren Lebewesen auch noch als sauber zu bezeichnen ist. Das Licht der Sonne erfüllt alle diese Bedingungen. Dem entsprechend prognostiziert der Wissenschaftliche Beirat Globale Umweltveränderungen WBGU der deutschen Bundesregierung bis zum Ende des 21. Jahrhunderts eine Zunahme des Anteils an Solarenergie beim globalen Energiemix auf bis ca. 60\%.[3]

Photoprozesse, d.h. Phänomene mit Lichtbeteiligung, sind Schlüsselvorgänge in Funktionseinheiten von größeren Systemen inder Naturund in derTechnik. So können beispielsweise das Blatt, das Auge, die Atmosphäre, eine Solarzelle, eine LED und viele ähnliche Systeme, bei denen Licht entweder als input oder als output beteiligt ist, als Photoreaktoren angesehen wer- den. Die aktuellen Anwendungen und die Forschung im Bereich der Photoprozesse erstrecken sich quer über alle naturwissenschaftlichen Disziplinen, die Material-, Lebens- und Ingenieurwissenschaften. In fachwissenschaftlichen Zeitschriften ist die Häufigkeit von Stichwörtern wie „Solarzellen“, „Energieumwandlung“, „Photochemie“, ,Photokatalyse“, ,Wasserphotolyse“ „Photochromie“, „Photosynthese“, „Photoaktive molekulare Schalter", „Lumineszenz" etc. außerordentlich hoch, mehrere Nobelpreise der letzten Jahre wurden für wissenschaftliche Leistungen vergeben, die mit solchen Stichwörtern zu tun haben.

DerVorrang von Photoprozessen bei der Entwicklung von hightech Materialien und Verfahren sowie von wissenschaftlichem und technischem know how als Garanten für Prosperität und Nachhaltigkeit in der Zukunft steht außer Zweifel. Daher müssen Photoprozesse im 21. Jahrhundert auch in der naturwissenschaftlichen Bildung, speziell im Chemieunterricht und in benachbarten MINT-Fächern, ein höheres Gewicht erhalten (Abb.1).

Um das $\mathrm{zu}$ beschleunigen, werden entsprechende Lehr-/Lerninhalte didaktisch erschlossen. Neue Fachinhalte zu Photoprozessen didaktisch erschließen heißt

1. überzeugende Experimente und Experimentreihen zu diesen Fachinhalten entwickeln,

2. sie mit wissenschaftlich konsistenten Konzepten und Modellen untermauern und

3. diese in die chemische Fachsystematik einbinden.

Die curriculare Innovationsforschung folgt diesem Fahrplan. ${ }^{[4,5]}$ Neben Experimenten und Konzepten liefert sie didaktische Materialien für Unterricht, Lehre und individuelles Lernen. Diese umfassen 


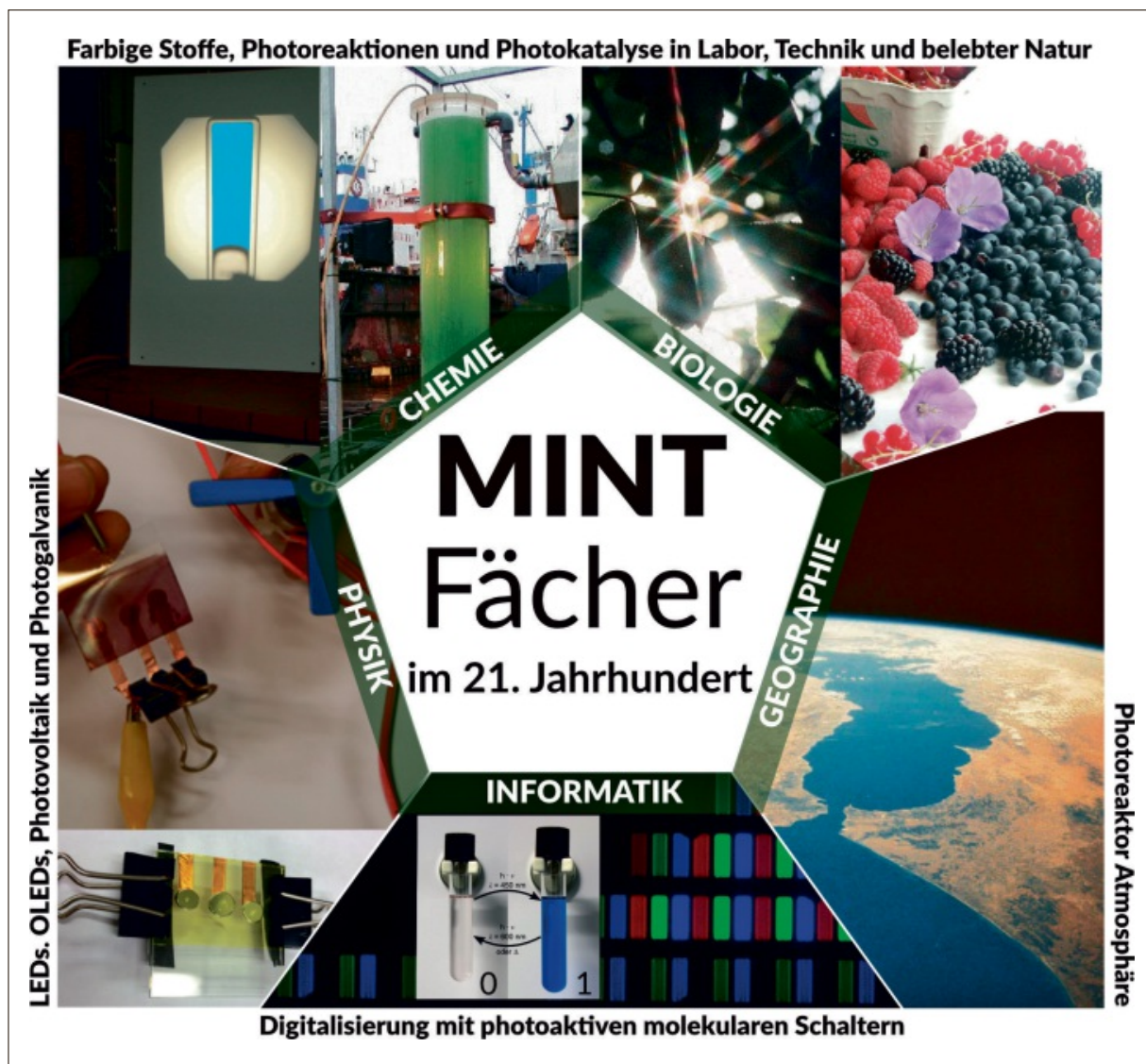

Abb. 1. Photoprozesse haben in den MINT-Fächern des Schulunterrichts Querschnittscharakter (Bildbearbeitung: Nico Meuter).

Printmedien vom Arbeitsblatt bis zum kompletten Lehrbuch sowie elektronische Medien vom Kurzvideo bis zum TutorialLehrfilm und von der Modellanimation bis zum Hypermedia-Netzwerk. Zeitgemäß und von großem Vorteil ist es, wenn die gedruckten und die elektronischen Materialien miteinander vernetzt sind und sich gegenseitig ergänzen. Über die Notwendigkeit, photochemische Inhalte in die Chemielehrerausbildung zu integrieren wird in diesem Heft an anderer Stelle berichtet (vgl. M. W. Tausch, Chimia 2018 , $72,13)$.

\section{Didaktisches Schlüsselkonzept und Klassifikation von Photoprozessen}

Für die Vermittlung photochemischer und photophysikalischer Inhalte im Schulunterricht enthält das folgende Zitat von N. J. Turro ${ }^{[6]}$ gleich drei grundsätzliche Gedanken:

,The 'photo' part of molecular photochemistry is a historical prefix and is now too restrictive. It is now clear that electronically excited states of molecules are the heart of all photoprocesses. The excited state is in fact an electronic isomer of the ground state."
Der erste Gedanke, die Metapher von den elektronisch angeregten Zuständen als „Herz“ aller Photoprozesse, also aller Phänomene mit Lichtbeteiligung, steht in gutem Einklang mit dem in den Lehrplänen aller Bundesländer in Deutschland verankerten Energiestufen-Modell für Moleküle. Das ist zwar eine recht grobe Vereinfachung des Jablonski-Diagramms für die Energiezustände in Molekülen, ${ }^{[1,7]}$ aber damit lassen sich alle Photoprozesse in erster Näherung wissenschaftlich konsistent erklären.

Der zweite insbesondere für die Didaktik des Chemieunterrichts und der Lehre der Chemie an Universitäten ebenfalls bemerkenswerte Gedanke, betrifft den elektronisch angeregten Zustand, den Turro als ein ,elektronisches Isomer“" des Grundzustandes bezeichnet. Wir haben es bei einem elektronisch angeregten Zustand tatsächlich mit einer anderen chemischen Spezies zu tun, die ganz andere Eigenschaften hat als das Molekül im Grundzustand, aber immer noch aus den gleichen Atomen besteht. Es handelt sich also schulmäßig um Isomere.

Die dritte, für Lehrende und Lernende ebenso wichtige Idee, steht zwar nicht explizit im Turro-Zitat, folgt aber aus dem ersten Satz darin: Der elektronisch angeregte Zustand A* eines Moleküls A muss nicht notwendigerweise durch Absorption eines Lichtquants erzeugt werden (Abb. 2). A* kann auch direkt aus einer stark exergonischen Reaktion hervorgehen (z.B. bei der Chemolumineszenz) oder durch Zufuhr von elektrischer Energie erzeugt werden (z.B. bei organischen Leuchtdioden).

Was das Abklingen oder die Desaktivierung von $\mathrm{A}^{*}$ anbetrifft, ergibt sich ein ganzer Zoo von Möglichkeiten (Abb. 2). Sie betreffen die unterschiedlichen Arten von Lumineszenz (Fluoreszenz, Phosphoreszenz, Chemolumineszenz, Elektrolumineszenz, Elektrochemolumineszenz), unterschiedliche Typen von photovoltaischen Zellen (z.B. anorganische Zellen mit $\mathrm{TiO}_{2}$ und organische mit Nano- $\mathrm{C}_{60}$ und leitenden Polymeren), Energietransfer von $A^{*}$ auf D (z. B. vom akzessorischen Pigment $\beta$-Carotin auf den Photokatalysator Chlorophyll), Isomerisierungen (z. B. E/ZIsomerisierungen), Photoredoxreaktionen (vgl. Teil 3 dieses Aufsatzes) und weitere Photoreaktionen. ${ }^{[8]} \mathrm{Zu}$ allen in Abb. 2 aufgezeigten Desaktivierungsmöglichkeiten des angeregten Zustands $A^{*}$ gibt es bereits Experimente und weiteres didaktisches Material aus eigener Forschung und Entwicklung. ${ }^{[9]}$ Diese Artikel sind auf der Website ${ }^{[7]}$ unter „Publikationen“ als open access verfügbar.

\section{Photo-Blue-Bottle - ein Experiment mit vielen Facetten}

Mit nur drei wasserlöslichen, toxikologisch harmlosen Chemikalien und LED-Taschenlampen oder Sonnenlicht lassen sich verschiedene Versionen des Photo-Blue-Bottle Experiments durchführen (Abb. 3). Es kann je nach Bedarf als Modellexperiment für Stoffkreisläufe, Redoxreaktionen, Energetik chemischer Reaktionen, galvanische Zellen, Katalyse und Relation TeilchenstrukturStoffeigenschaften funktionalisiert werden. Dabei sind diese allgemeinen Konzepte der Chemie ebenso wie die Bezeichnung des Experiments selbst jeweils mit dem Präfix Photo zu verknüpfen.

Das Experiment wurde über einen Zeitraum von mehr als zwanzig Jahren getestet, optimiert und in den Lehrgang CHEMIE 2000+ eingebunden. ${ }^{[10]}$ Die in Abb. 3 dargestellten low cost Versionen sind im Rahmen des DFG-Projekts Photo-LeNa (Photoprozesse in der Lehre der Naturwissenschaften) in einem Promotionsvorhaben entwickelt worden. ${ }^{[11,12]}$ Das Equipment für diese Versuche kann als Experimentierbox über die Wuppertaler Website unter dem Link http://www.chemiedidaktik.uniwuppertal.de/lehre/photo-cat/index.html bezogen werden. Dort sind auch Infos mit fachlichen Grundlagen, didaktischen 
Abb. 2. Erzeugung und Desaktivierungsmöglichkeiten des elektronisch angeregten Zustands $A^{*}$ (F: Fluoreszenz, $P$ : Phosphoreszenz, CL: Chemolumineszenz, EL: Elektrolumineszenz, ECL: Elektrochemolumineszenz).

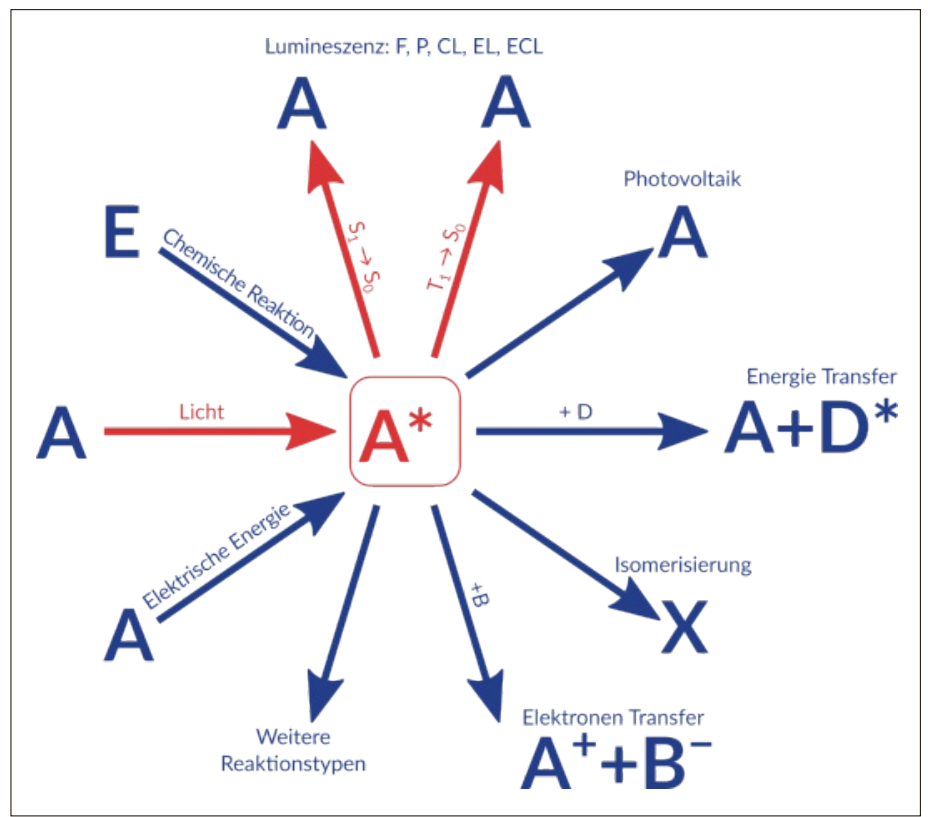

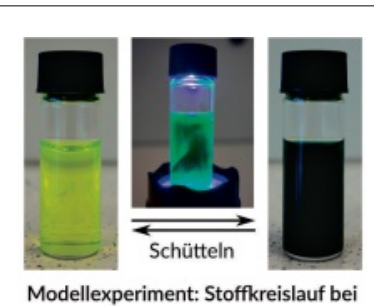

Modellexperiment: Stoffkreislauf be der Photosynthese und Zellatmung<smiles>Nc1ccc2cc3ccc(N)cc3nc2c1</smiles>

Photokatalysator Proflavin

3,6-Diaminoacridin-hemisulfat

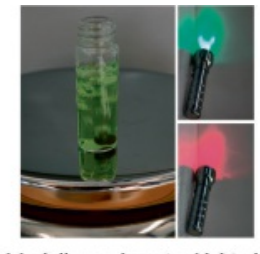

Modellexperimente: Licht als Antrieb für die Photosynthese

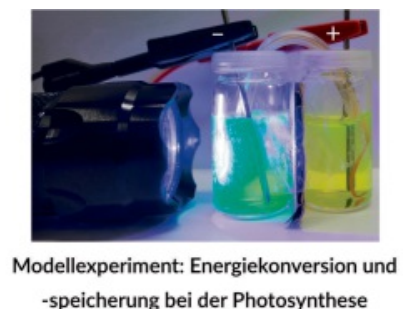

-speicherung bei der Photosynthese<smiles>O=C(O)CN(CCN(CC(=O)O)CC(=O)O[Na])CC(=O)O</smiles>

Opferdonator EDTA

Ethylendiaminotetraessigsäuredinatriumsalz

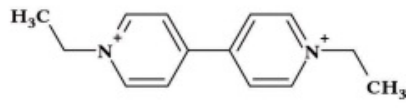

Substrat

Ethylviologen $\mathrm{EV}^{++}$ 1,1'-Diethyl-4,4'-bipyridiniumdibromid

Abb. 3. Drei Versionen, didaktische Funktionen und Chemikalien des Photo-Blue-Bottle Experiments (Bildbearbeitung: Nico Meuter).

Hinweisen, Arbeitsblätter und der Lehrfilm „Photosynthese - ein Fall für zwei“ kostenlos zum Ansehen und Herunterladen bereitgestellt. Mit dem Benutzernamen „Tausch“ und dem Passwort „Photo-Cat“ können bereits publizierte Artikel aus der Literatur als pdf-Dateien geöffnet werden. Dank dieser umfangreichen, leicht zugänglichen online Dokumentation können an dieser Stelle die wichtigsten Phänomene und die damit verbundenen Elementarprozesse wie folgt zusammengefasst werden: Die gelbe Lösung färbt sich bei Bestrahlung mit Sonnenlicht oder mit blauem Licht einer LED-Taschenlampe blau. Dabei wird das EthylviologenDikation $\mathrm{EV}^{++}$zum Monokation $\mathrm{EV}^{+}$reduziert (Abb. 3). Das ist nur möglich, weil die
Molekül-Ionen $\mathrm{PF}^{+}$des Photokatalysators Proflavin durch Photonen aus dem blauen Spektralbereich in den elektronisch angeregten Zustand $\mathrm{PF}^{+*}$ angeregt werden und von dort jeweils ein Elektron an ein Dikation $\mathrm{EV}^{++}$übertragen wird (vgl. Abb. 4, Photoelektronentransfer). Ein Teil der Energie des absorbierten Photons ist jetzt im reduzierten Substrat $\mathrm{EV}^{+}$gespeichert.

Dass die Reduktion des Substrats nicht thermisch und auch nicht mit grünem oder rotem Licht angetrieben werden kann, wird mit der in Abb. 3 (Mitte) dargestellten Version des Photo-BlueBottle Experiments erarbeitet. Der Effekt der Energiekonversion und -speicherung erfolgt in der photoelektrochemischen Konzentrationszelle aus dem rechten Teil in Abb. 3. Die zusammenfassende Gegenüberstellung in Abb. 5 hebt einige Analogien zwischen dem Modellexperiment und dem natürlichen Stoffkreislauf bei der Photosynthese und Atmung hervor.

Diese Analogien, aber auch die Unterschiede zwischen dem Modellexperiment und den Vorgängen in der Natur bieten gute Möglichkeiten für kritische Reflexion in forschend-entwickelndem Chemie- und Biologieunterricht.

\section{Ausblick}

Im laufenden DFG-geförderten Forschungsprojekt Photo-MINT wird die Qualitätssteigerung der Lehre im Fach Chemie und den benachbarten MINTFächern durch curriculare Innovation angestrebt. Der fachliche und didaktische Mehrwert für die Lehre fokussiert als Leitmotiv den elektronisch angeregten Zustand $\mathrm{A}^{*}$ von Molekülen und anderen Teilchenverbänden (vgl. Teil 2 dieses Beitrags). Die möglichen Wege für seine Erzeugung und Desaktivierung (vgl. Abb. 2) werden aus didaktischer Perspektive erschlossen, strukturiert, mit Experimenten, Konzepten, Print- und Elektronikmaterialien für Unterricht und Lehre ausgestattet und in der Lehrerausbildung und -fortbildung vermittelt. Dem interdisziplinären Charakter der Phänomene mit Lichtbeteiligung soll dabei besondere Beachtung geschenkt werden. Aus didaktischer Sicht ist es für jedes der Fächer aus Abb. 1 gewinnbringend, wenn die für das jeweilige Fach relevanten stofflichen und energetischen Phänomene bei der Wechselwirkung Licht-Materie auf die Elementarprozesse bei und nach der Interaktion von Photonen mit Molekülen (oder anderen Teilchenverbänden) zurückgeführt werden, also auf diskrete Einheiten, wie sie für die chemische Betrachtungsweise charakteristisch sind. Insofern erhält die Chemie der angeregten Zustände bei der Erschließung und curricularen Integration von Photoprozessen in die Lehrgänge anderer MINT-Fächer eine wegweisende Funktion. Diese Arbeitshypothese wird in mehreren Modulen konkretisiert, die auf jeweils einem oder mehreren Experimenten aus Abb. 6 basieren. In Kooperation zwischen den Fachdidaktiken aus Wuppertal, Köln, Münster und Tübingen wurden bereits die Materialienkoffer „Photo-Mol““[13] und „Organic Photo Electronics“[14] nach dem Format von Interaktionsboxen entwickelt, die in Teil 3 erwähnte Materialienbox „Photo-Cat" ist in Erprobung und an einer weiteren Experimentreihe, die zur „Photo-Switsch“ Box führen soll, wird geforscht. 


\section{Danksagung}

Der Deutschen Forschungsgemeinschaft DFG wird für die Förderung der Forschungsvorhaben TA 228/4-1 Photo-LeNa (Photoprozesse in der Lehre der Naturwissenschaften, 2013-2016) und TA 228/4-2 PhotoMINT (Photoprozesse in den MINT-Fächern, 2017-2020) gedankt.

Received: October 19, 2017

[1] D. Wöhrle, W.-D. Stohrer, M. W. Tausch, 'Photochemie, Konzepte, Methoden, Experimente', Wiley-VCH, Weinheim, 1998.

[2] M. Beller, G. Centi, D. Nocera, G. M Kemeling, P. Göltz: 'Editorial: Foodsteps on the Sustainability Trail', ChemSusChem 2010, 3, 3; D. Nocera: 'The Sustainocene - era of personalized energy', video auf youtube unter: https:// www.youtube.com/watch $? v=u 9208$ LskezY, letzter Zugriff: 19.10.2017.

[3] http://www.agenda21-treffpunkt.de/archiv/04/ daten/WBGUenergiemix.htm, letzter Zugriff: 19.10.2017.

[4] M. W. Tausch, A. Flint, 'Experimentell-Konzeptionelle Forschung in der Chemiedidaktik', Nachrichten aus der Chemie 2016, 64, 359.

[5] M. W. Tausch, 'Curriculare Innovationsforschung in der Chemiedidaktik', Praxis der Naturwissenschaften - Chemie in der Schule, 2013, 62 (4), 38.

[6] N. J. Turro, 'Modern Molecular Photochemistry', Benjamin/Cummings, New York, 1978 (erste Auflage), University Science Books, Sausalito, 1991 (neueste Auflage).

[7] www.chemiedidaktik.uni-wuppertal.de, Experimente, Videos, Lehrfilme.

[8] M. W. Tausch, 'Photoprozesse in der Lehre der Naturwissenschaften', Praxis der Naturwissenschaften - Chemie in der Schule, 2015 64 (1), 5.

[9] M. W. Tausch (Hrsg.), Praxis der Natur wissenschaften-Chemie in der Schule, Themenheft 'All We Need Is Light', 2015, 64 (1) und Themenheft 'Funktionelle Farbstoffe', 2016, 65 (1).

[10] M. Tausch, M. von Wachtendonk (Hrsg.), 'CHEMIE 2000+, Sekundarstufe II', C.C. Buchner Verlag, Bamberg, 2007.

[11] M. W. Tausch, M. Heffen, 'Photokatalyse - homogen und heterogen, Das Photo-BlueBottle Experiment runderneuert', Praxis der Naturwissenschaften - Chemie in der Schule $\mathbf{2 0 1 5}, 64(8), 42$

[12] M. W. Tausch, M. Heffen, 'Photosynthese und Atmung en miniature - Teil 1', Chemie \& Schule 2016, 31 (3), 5

[13] M. W. Tausch, N. Meuter, 'Funktionelle Farbstoffe - Interaktionsbox für Schulen und Universitäten', Praxis der Naturwissenschaften - Chemie in der Schule 2016, 65 (1), 5.

[14] A. Banerji, „Organische Elektronik als Lehrstoff", Nachrichten aus der Chemie 2017, $65,807$.

[15] M.W. Tausch et al. Lehrfilme zu Photoprozessen unter: http://www.chemiedidaktik.uni-wuppertal.de/lehre/lehrfilme/index.html

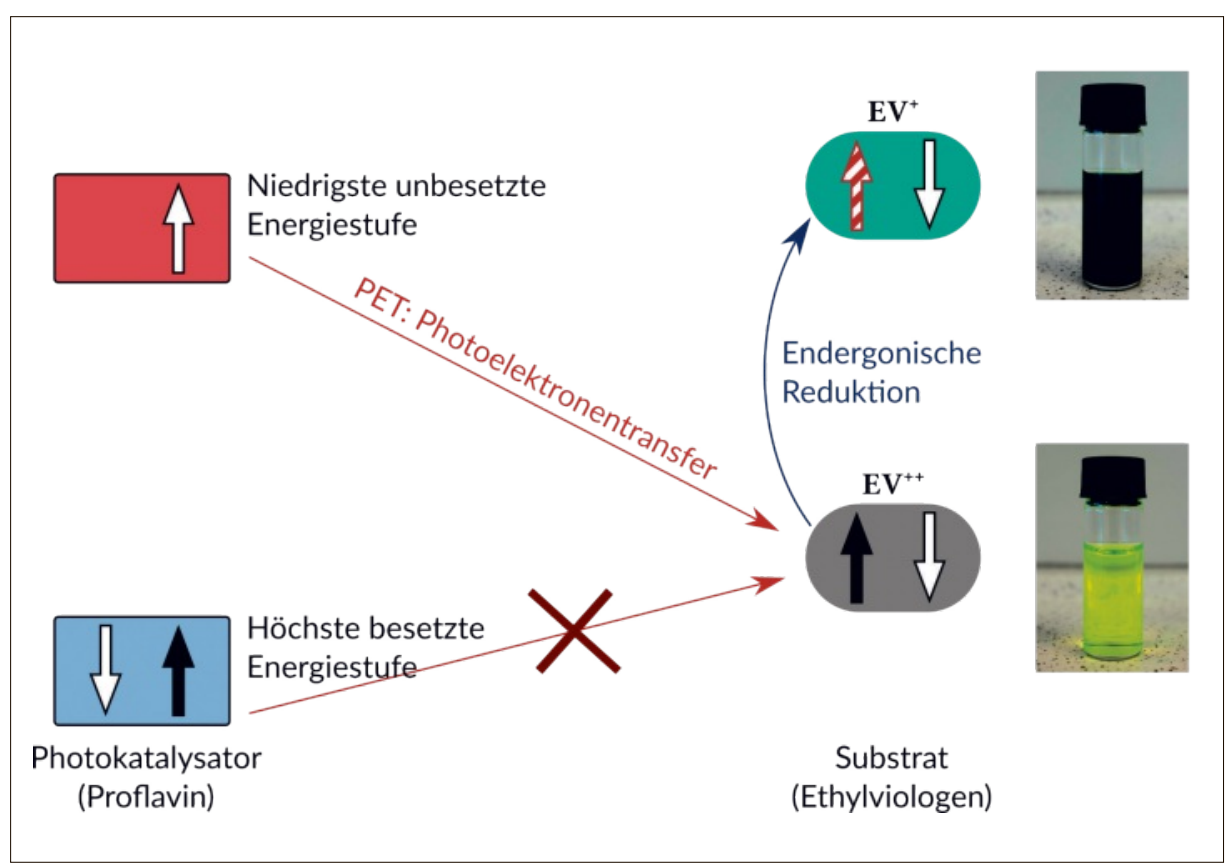

Abb. 4. Elektronische Anregung und Elektronentransfer bei der photokatalytischen Reduktion von Ethalviologen im Photo-Blue-Bottle Experiment (Bildbearbeitung: Nico Meuter).

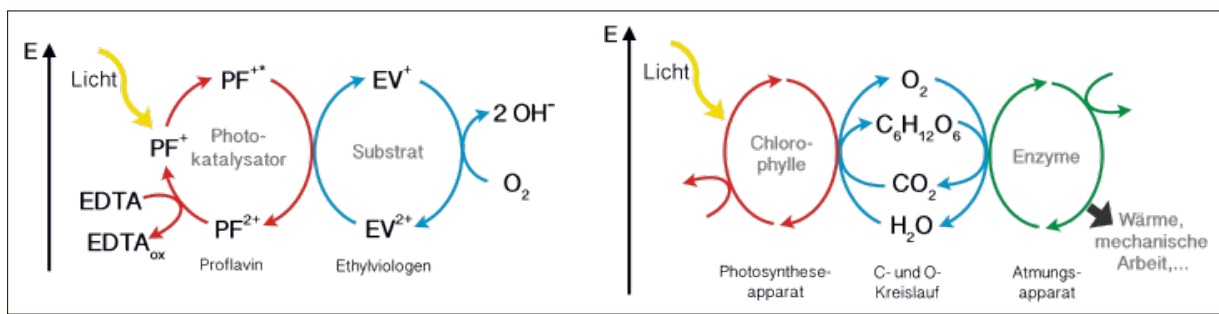

Abb. 5. Die gekoppelten Reaktionszyklen beim Photo-Blue-Bottle Experiment und beim natürlichen Stoffkreislauf Photosynthese-Zellatmung laufen in wässriger Lösung unter Beteiligung von farbigen Stoffen und Sauerstoff aus der Luft ab. Sie werden durch sichtbares Licht angetrieben.

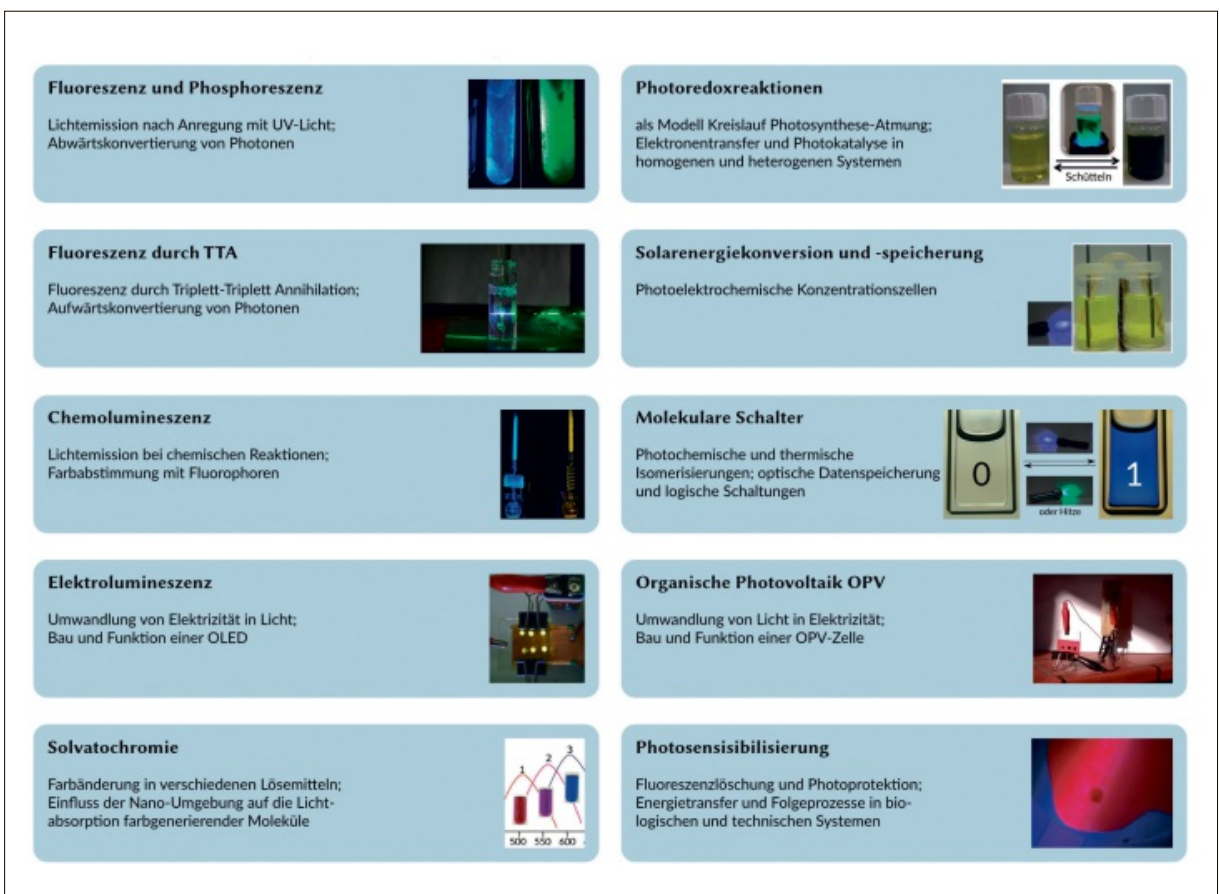

Abb. 6. Die top ten Experimente der curricularen Innovationsforschung in Wuppertal (Bildbearbeitung: Nico Meuter). 